\title{
The effect of attentional demands on the antisaccade cost
}

\author{
Richard GodiJn AND ARTHUR F. KRAMER \\ Beckman Institute, University of Illinois at Urbana-Champaign, Urbana, Illinois
}

\begin{abstract}
In the present study, we examined the effect of attentional demands on the antisaccade cost (the latency difference between antisaccades and prosaccades). Participants performed a visual search for a target digit and were required to execute a saccade toward (prosaccade) or away from (antisaccade) the target. The results of Experiment 1 revealed that the antisaccade cost was greater when the target was premasked (i.e., presented through the removal of line segments) than when it appeared as an onset. Furthermore, in premasked target conditions, the antisaccade cost was increased by the presentation of onset distractors. The results of Experiment 2 revealed that the antisaccade cost was greater in a difficult search task (a numeral 2 among $5 \mathrm{~s}$ ) than in an easy one (a 2 among 7s). The findings provide evidence that attentional demands increase the antisaccade cost. We propose that the attentional demands of the search task interfere with the attentional control required to select the antisaccade goal.
\end{abstract}

Human behavior can be characterized by its flexibility in responding to events in our environment. A classic demonstration of this flexibility was provided in a study by Hallett (1978; see also Hallett \& Adams, 1980). Under normal circumstances, observers execute saccadic eye movements (saccades) toward objects that are of interest to their goals and desires. However, Hallett demonstrated that when observers were instructed to move their eyes in the opposite direction from a stimulus, they were able to execute such antisaccades on the vast majority of trials. In the original version of the task, participants were instructed to fixate a stimulus presented at the center of the screen. The stimulus abruptly stepped to the left or right of fixation, and participants were required to move their eyes in the direction opposite from the step (antisaccade). In another version of this antisaccade task (typically referred to as the overlap condition), there is no movement of a fixation stimulus, but a peripheral stimulus appears while the central fixation point remains on the screen. Participants were quite good at executing antisaccades, but on some trials they erroneously moved their eyes toward the stimulus (erroneous prosaccade), despite the instruction to move their eyes in the opposite direction.

Hallett and Adams (1980) developed a model of antisaccade generation in which a prosaccade program is automatically initiated. If the prosaccade program is completed before an antisaccade goal redefinition program is initiated, a prosaccade is executed. If the antisaccade goal redefinition process is initiated prior to the completion of the prosaccade program, that program is canceled, and an antisaccade is executed instead. Subsequently, Guitton, Buchtel, and Douglas (1985) found that patients with frontal-lobe damage executed a higher percentage of erroneous prosaccades in the antisaccade task than did a control group (see also Milea et al., 2003; Pierrot-Deseilligny, Ploner, Müri, Gaymard, \& Rivaud-Péchoux, 2002; PierrotDeseilligny, Rivaud, Gaymard, \& Agid, 1991; Walker, Husain, Hodgson, Harrison, \& Kennard, 1998). Utilizing the Hallett and Adams model, they proposed that frontal-lobe damage delays the cancellation signal (or the goal redefinition process) such that, on many trials, it occurs too late to stop the execution of the prosaccade program.

Since these early studies, the antisaccade task has become one of the most popular eye movement paradigms. Its use has extended beyond behavioral and clinical studies, to developmental, human neuroimaging, and monkey neurophysiological studies (for a review, see Munoz \& Everling, 2004). Given the popularity of the antisaccade task, as well as the ubiquitous nature of eye movements in most everyday behaviors, it is obvious that an understanding of the processes involved in antisaccade generation is of great importance.

Following the initial steps of Hallett and Adams (1980), more recent studies have further examined the processes involved in antisaccade generation (e.g., Kristjánsson, Chen, \& Nakayama, 2001; Kristjánsson, Vandenbroucke, \& Driver, 2004; Massen, 2004; Olk \& Kingstone, 2003). Typically, performance in the antisaccade task is compared with performance in a prosaccade task. It is consistently found that more saccade errors occur in the antisaccade task (prosaccade errors) than in the prosaccade task. Furthermore, an antisaccade cost is also found consistently; that is, saccade latencies are longer in the antisaccade than in the prosaccade task. Although the processes involved

R. Godijn, rj.godijn@psy.vu.nl 
in the antisaccade task remain disputed, it is commonly held that in this task a reflexive prosaccade is automatically programmed and that correct antisaccade generation requires the inhibition of the reflexive prosaccade (e.g., Everling \& Munoz, 2000; Olk \& Kingstone, 2003; Pratt \& Trottier, 2005).

For example, Olk and Kingstone (2003) showed that when the correct saccade goal (pro- or antisaccade) was determined by the orientation of a peripheral arrow, the antisaccade cost was reduced. Since participants in the prosaccade condition had to attend to the peripheral arrow in order to determine the correct saccade goal, it was assumed that they inhibited a prosaccade while processing the orientation of the arrow. The finding that this manipulation reduced the antisaccade cost was interpreted as evidence that the antisaccade cost is largely due to reflexivesaccade inhibition. This view presumes that processes in the antisaccade task are serial; that is, inhibiting a reflexive prosaccade is time-consuming and precedes the programming of the antisaccade.

An alternative view was recently proposed by Massen (2004). In a series of experiments, she demonstrated that manipulations that increased antisaccade latencies also increased the percentage of erroneous prosaccades in the antisaccade task, whereas manipulations that increased prosaccade latency reduced the percentage of erroneous prosaccades in the antisaccade task. For example, in Experiment 1 of her study, participants were required to execute a pro- or antisaccade on the basis of the orientation of a central cue, and the relative frequency of antisaccades was varied between blocks. The results showed that, whereas prosaccade latencies were unaffected by the probability manipulation, there was a negative relationship between antisaccade latency and antisaccade frequency. Thus, as antisaccade frequency decreased, the programming time of antisaccades increased, while that of prosaccades remained constant. Furthermore, slowing down the antisaccade resulted in an increase in the proportion of erroneous prosaccades in the antisaccade condition. To account for her findings, Massen proposed a model of antisaccade generation in which a reflexive prosaccade and an antisaccade are programmed independently and in parallel. The saccade program that first reaches a threshold is the one executed.

However, several findings from Kristjánsson and colleagues (Kristjánsson, 2007; Kristjánsson et al., 2001; Kristjánsson et al., 2004) have provided evidence for the view that prosaccades and antisaccades are not programmed independently, but instead compete interactively. For example, Kristjánsson et al. (2001) showed that when attending to a secondary task (a peripheral discrimination task) shortly before the presentation of the antisaccade stimulus, latencies were increased in the prosaccade task but reduced in the antisaccade task. Kristjánsson et al. (2001) interpreted this finding as evidence for a competition account of antisaccade generation (see also Kristjánsson, 2007). They proposed that the secondary task interfered with the reflexive prosaccade, so that fewer attentional resources were needed for suppression of the prosaccade, thereby leaving more attentional resources for the selection of the antisaccade goal. In a follow-up study, Kristjánsson et al. (2004) demonstrated that certain manipulations had opposite effects on prosaccades and antisaccades, which provided further evidence for the competition account of antisaccade generation.

Despite the differences between current views on antisaccade generation (e.g., Kristjánsson, 2007; Massen, 2004; Olk \& Kingstone, 2003), they all share the assumption that reflexive prosaccades are programmed in the antisaccade task; performance in the antisaccade task is seen as a race or competition between a reflexive or exogenous saccade and a voluntary or endogenous saccade. Intuitively this makes sense, because the prosaccade stimulus is typically salient, prosaccade latencies are relatively short, and in the antisaccade task a prosaccade violates the task instructions. However, it should be noted that the antisaccade stimulus is task-relevant; that is, participants are required to localize the antisaccade stimulus in order to determine the correct saccade goal. In fact, we have recently provided evidence for an endogenous component to prosaccades (Godijn \& Kramer, 2006). We examined the nature of erroneous prosaccades in a task in which participants viewed displays containing three gray circles presented on an imaginary square around a central fixation location. On each trial, two of the three circles turned red, leaving a uniquely colored gray circle (the color singleton). Simultaneously with this color change, a new red circle appeared elsewhere on the imaginary circle. In some blocks, participants were required to search for the onset; on others, they were required to search for the color singleton. In half of the blocks, the task was to execute a prosaccade toward the search target, and in the other half it was to execute an antisaccade in the direction opposite the search target. We found that when the onset or the color singleton was the search target in the antisaccade condition, the percentage of erroneous saccades in their direction ranged from $10 \%$ to $18 \%$, but when they were task-irrelevant (i.e., presented as the distractor), the eyes moved in their direction on less than $3 \%$ of the trials. These results provide evidence that task relevance is an important factor in the execution of erroneous prosaccades.

In a follow-up study, we examined the antisaccade cost with static and dynamic search targets (Godijn \& Kramer, 2007). Participants either searched for a unique dynamic target (an onset or offset) or a unique static target (a color singleton) and executed a prosaccade or antisaccade in response to the location of the target. We consistently found that the antisaccade cost was greater for static than for dynamic targets. Furthermore, the percentage of erroneous prosaccades in the antisaccade conditions was higher with static than with dynamic targets. Clearly, the erroneous prosaccades in these experiments could not be considered reflexive (see also Godijn \& Kramer, 2006). Moreover, the antisaccade cost was smaller in the conditions in which the reflexive pull toward the search target was expected to be greater (the dynamic search targets).

These recent findings from our lab (Godijn \& Kramer, 2006,2007 ) provide evidence that erroneous prosaccades are not entirely reflexive and that a substantial antisaccade cost is found when there is no reflex to inhibit (static tar- 
gets). One possible explanation for these counterintuitive findings is that the attentional demands of localizing the prosaccade target compete with those of selecting the antisaccade goal. Indeed, there is substantial evidence that attention is required in order to program endogenous saccades (e.g., Deubel \& Schneider, 1996; Godijn \& Pratt, 2002; Hoffman \& Subramaniam, 1995; Kowler, Anderson, Dosher, \& Blaser, 1995). If attention is allocated at the prosaccade location in order to localize the target, but attention is required at the antisaccade location, the pro- and antisaccade locations compete for attentional resources.

The goal of the present study was to examine the effect of attentional demands on the antisaccade cost. If the attentional demands of localizing the prosaccade target compete with the attentional selection of the antisaccade location, one would expect increasing the attentional demands of target localization to increase the antisaccade cost. That is, the attentional demands would be expected to have a greater effect on antisaccade than on prosaccade latencies.

We examined the effect of attentional demands on prosaccades and antisaccades utilizing two attentional manipulations. In both experiments, participants performed a visual search for a target digit (a 2), which was presented together with homogeneous distractor digits $(5 \mathrm{~s}$ or $7 \mathrm{~s})$. In Experiment 1, digits were either presented as onsets or by the removal of line segments from a figure-eight premask. Previous research established that onsets capture attention in a stimulus-driven manner (e.g., Theeuwes, 1991; Yantis, 1996). Therefore, if the attentional demands of the search task compete with the attentional selection of the antisaccade goal, the antisaccade cost should be greater for premasked targets than for onset targets. Furthermore, when the target is premasked, the antisaccade cost is expected to be larger when onset distractors are presented than when all digits are premasked. That is, the onset distractors are expected to capture attention, thereby disrupting target localization and increasing the attentional demands. In Experiment 2, participants performed either an easy search (a 2 among $7 \mathrm{~s}$ ) or a difficult one (a 2 among 5s). If the attentional demands of the search task compete with the attentional selection of the antisaccade goal, the antisaccade cost is expected to be greater in the difficult than in the easy search task, since the attentional demands are higher in the difficult search task (see Wolfe \& Horowitz, 2004, for a review of the stimulus attributes that guide attention). If the attentional demands of the search task do not compete with the attentional selection of the antisaccade goal, the attentional manipulations of both experiments would be expected to affect both pro- and antisaccade latencies equally. Specifically, increasing the attentional demands of the search task should increase both pro- and antisaccade latencies to the same extent.

\section{EXPERIMENT 1}

\section{Method}

Participants. After giving informed consent, 22 students from the University of Illinois with normal or corrected-to-normal vision served as paid volunteers.

Apparatus. A Pentium-based computer with a 21-in. color monitor controlled the timing of events and generated stimuli. Eye move- ments were recorded by means of an Eyelink II eyetracker. An eye movement was considered a saccade when the velocity exceeded $35^{\circ} / \mathrm{sec}$ or the acceleration exceeded $9,500^{\circ} / \mathrm{sec}^{2}$. When participants were fixating the central fixation point at the start of each trial, they pressed a key, which caused a recalibration of the participants' gaze point to the central fixation point. After this recalibration procedure, the trial started. Each participant was tested in a dimly lit room. Their heads were held on a chinrest located $70 \mathrm{~cm}$ from the monitor.

Stimuli, Procedure, and Design. At the start of each trial, participants viewed displays (the fixation display) containing six, four, two, or zero white figure-eight premasks $\left(0.6^{\circ} \times 1.2^{\circ}\right)$, which were randomly presented at six possible object locations around a central white fixation point $\left(0.2^{\circ}\right)$ at an eccentricity of $7.6^{\circ}$ on a black background. Two of the six possible object locations were directly above and below the central fixation point, and the four other possible locations were the corner positions of an imaginary square around the fixation point. After $1,000 \mathrm{msec}$, the fixation dot changed into a fixation cross, signaling the onset of the search target display. At the same time, line segments were removed from the figure-eights to reveal digits, while additional (onset) digits appeared at the unfilled locations. One of the digits was a numeral 2 (the search target), and the other five were 7s (the distractors). See Figure 1 for an illustration of the display sequence. The locations of the premasks, the number of premasks, and the location of the search target were randomized within blocks. The target was equally likely to appear at any of the six locations. The search display was removed $500 \mathrm{msec}$ after the correct saccade. The participants performed two blocks of 324 trials, which were both preceded by 20 practice trials. In one block, participants were instructed to execute a prosaccade toward the location of the search target, and in the other they were instructed to execute an antisaccade to the location opposite the search target. The order of the blocks was randomized across participants.

\section{Results}

Data analysis. We conducted analyses on the two main measures of performance in the prosaccade and antisaccade task: the percentage of erroneous prosaccades and mean latencies of correct saccades. Whereas there are typically very few errors in the prosaccade task (at least in the standard prosaccade task; see, e.g., Hallett \& Adams, 1980), in the antisaccade task many saccades are erroneously directed toward the prosaccade location. Since the percentage of erroneous prosaccades in the antisaccade task can provide insights into the competition between the pro- and antisaccade locations, the error analyses in this study focused on this measure. Our other main measures were the mean latencies of correct saccades in the prosaccade task and the antisaccade task. In particular, the antisaccade cost (i.e., the difference in mean latencies between correct prosaccades and antisaccades) was examined, since we hypothesized that the attentional manipulations would have a greater effect on antisaccade than on prosaccade latencies. In addition, we examined the intersaccade interval (ISI) after erroneous prosaccades in order to determine the speed with which these errors were corrected.

Discarded data. Trials on which the initial saccade latency was below $100 \mathrm{msec}(5.5 \%)$ or above $1,200 \mathrm{msec}$ $(0.1 \%)$ were discarded from further analyses. Trials on which the first saccade had an amplitude of less than $2^{\circ}$ $(5.1 \%)$ were also discarded from the analyses.

Initial saccade destination. The distance between the initial saccade endpoint and the six possible object locations was used to determine the initial saccade destination. 


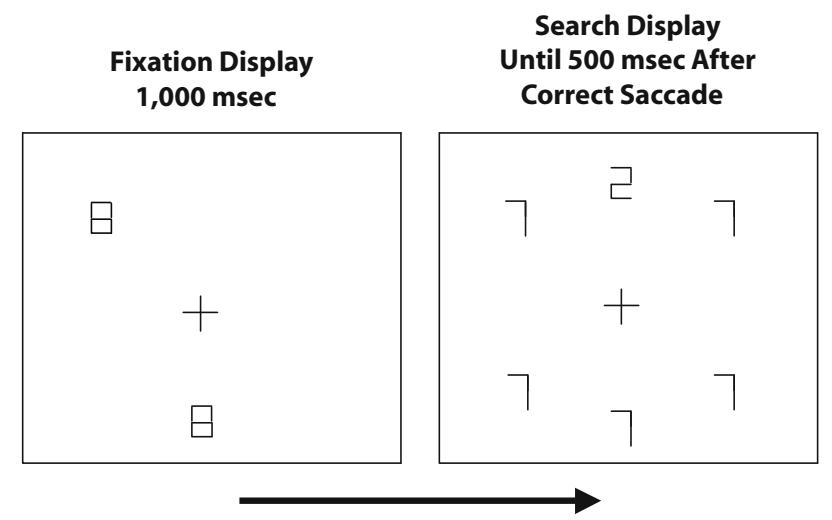

Figure 1. Example of the display sequence in Experiment 1. Shown is a trial on which two digits are premasked and four onsets are presented, one of which is the target (the 2). In separate blocks, participants were required to execute a prosaccade or an antisaccade in response to the target. See the text for further details.

The saccade was assigned to the position closest to the saccade endpoint. Table 1 shows the percentages of initial saccades that were directed to the prosaccade location, the antisaccade location, and the "other" locations, as a function of display type (number of onsets and premasks) and target type (onset or premask). In the antisaccade task, we compared the percentages of both erroneous prosaccades and erroneous saccades to "other" locations between the all-onset and all-premask conditions. The difference in erroneous prosaccades did not reach significance $[t(21)=$ $1.58, p>.10]$, but there was a significant difference in the percentage of saccades to "other" locations $[t(21)=3.89$, $p<.001]$. An ANOVA was then conducted on the percentages of erroneous prosaccades in the antisaccade condition, with display type (four premasks and two onsets vs. two premasks and four onsets) and target type (onset vs. premask) as factors. There was a main effect of target type $[F(1,21)=5.14, p<.04]$, indicating a higher percentage of erroneous prosaccades toward premask targets than toward onset targets. The effect of display type did not reach significance $[F(1,21)=3.11, p>.05]$, nor did the interaction between display type and target type $[F(1,21)=$ $2.52, p>.10]$. An additional ANOVA revealed that more erroneous saccades in the antisaccade task also occurred to "other" locations with a premask target than with an onset target $[F(1,21)=16.92, p<.001]$. This finding might suggest that the higher percentage of erroneous prosaccades with premask than with onset targets was due to a higher percentage of guesses with premask than with onset targets. However, further analyses revealed that in the antisaccade task, more erroneous saccades went to "other" onset distractors (i.e., onsets that were not at the pro- or antisaccade location) than to "other" premasked distractors $(8.4 \%$ vs. $6.2 \%)[t(21)=2.65, p<.02]$.

Saccade latencies. The mean latencies of correct prosaccades and antisaccades, as well as the antisaccade cost, are shown for each condition in Table 2. An ANOVA with target type (onset or premask) and task (prosaccade or antisaccade) as within-subjects factors, averaged across display type, was conducted on the mean latencies of the correct saccades. A main effect of target type was found $[F(1,21)=$ $126.96, p<.001]$, indicating that saccade latencies were longer when the target was premasked (mean $402 \mathrm{msec}$ ) than when it was an onset (mean $338 \mathrm{msec}$ ). There was also a main effect of task $[F(1,21)=152.07, p<.001]$, indicating that correct prosaccade latencies $(307 \mathrm{msec})$ were shorter than correct antisaccade latencies (433 msec). Most importantly, there was an interaction between target type and task $[F(1,21)=33.03, p<.001]$, which indicated that the antisaccade cost was greater for premasked targets than for onset targets. To examine the effect of onset distractors on the antisaccade task, we conducted separate ANOVAs

Table 1

Percentages of Initial Saccades to the Prosaccade Location (Pro), the Antisaccade Location (Anti), and Other Locations, As a Function of Display Type and Target Type in Experiment 1

\begin{tabular}{|c|c|c|c|c|c|c|c|c|}
\hline \multicolumn{2}{|c|}{ Display Type } & \multirow{2}{*}{$\begin{array}{l}\text { Target } \\
\text { Type }\end{array}$} & \multicolumn{3}{|c|}{ Prosaccade Task } & \multicolumn{3}{|c|}{ Antisaccade Task } \\
\hline Onsets & Premasks & & Pro & Anti & Other & Pro & Anti & Other \\
\hline 6 & 0 & Onset & 96.1 & 0.6 & 3. & 28.6 & 63.3 & 8.1 \\
\hline 0 & 6 & Premask & 83.9 & 2.2 & 13. & 23.9 & 62.2 & 13.9 \\
\hline \multirow[t]{2}{*}{4} & 2 & Onset & 96.4 & 0.7 & 2.9 & 22.7 & 67.0 & 10.3 \\
\hline & & Premask & 80.6 & 3.3 & 16.1 & 26.9 & 55.3 & 17.8 \\
\hline \multirow[t]{2}{*}{2} & 4 & Onset & 96.7 & 1.0 & 2.3 & 18.3 & 72.2 & 9.5 \\
\hline & & Premask & 74.0 & 4.2 & 21.8 & 26.5 & 52.9 & 20.6 \\
\hline
\end{tabular}


Table 2

Latencies of Correct Saccades (in Milliseconds) in the Pro- and Antisaccade Tasks As a Function of Display Type and Target Type in Experiment 1, As Well As the Antisaccade Cost (Difference in Latencies Between Correct Antisaccades and Correct Prosaccades)

\begin{tabular}{|c|c|c|c|c|c|c|c|}
\hline \multirow{2}{*}{\multicolumn{2}{|c|}{ Display Type }} & \multirow{3}{*}{$\begin{array}{l}\text { Target } \\
\text { Type }\end{array}$} & \multicolumn{4}{|c|}{ Task } & \multirow{3}{*}{$\begin{array}{c}\text { Antisaccade } \\
\text { Cost }\end{array}$} \\
\hline & & & \multicolumn{2}{|c|}{ Prosaccade } & \multicolumn{2}{|c|}{ Antisaccade } & \\
\hline Onsets & Premasks & & $M$ & $S E$ & $M$ & $S E$ & \\
\hline 6 & 0 & Onset & 280 & 10.9 & 394 & 14.2 & 114 \\
\hline 0 & 6 & Premask & 332 & 11.4 & 457 & 16.8 & 125 \\
\hline \multirow[t]{2}{*}{4} & 2 & Onset & 286 & 11.6 & 393 & 14.9 & 107 \\
\hline & & Premask & 329 & 13.0 & 473 & 18.2 & 144 \\
\hline \multirow[t]{2}{*}{2} & 4 & Onset & 284 & 11.3 & 389 & 15.6 & 105 \\
\hline & & Premask & 331 & 12.8 & 491 & 19.2 & 160 \\
\hline
\end{tabular}

for onset and premask targets, with task and display type as factors. For onset targets, no main effect of display type $[F(2,42)<1]$ and no interaction between display type and task $[F(2,42)=1.17, p>.30]$ emerged, indicating that the number of onset distractors (one, three, or five) did not significantly affect saccade latencies or the antisaccade cost when the target was an onset. However, for premask targets, both a main effect of display type $[F(2,42)=4.11$, $p<.03]$ and an interaction between display type and task $[F(2,42)=9.51, p<.001]$ appeared. Thus, the number of onset distractors (zero, two, or four) did have an effect on the antisaccade cost when the target was premasked. We examined this interaction by comparing the antisaccade costs of each pair of display types with premasked targets. The antisaccade cost (the interaction between task and display type) was greater both with two onset distractors $[F(1,21)=$ $5.49, p<.03]$ and with four $[F(1,21)=20.38, p<.001]$ than with no onset distractors. The difference between the two-distractor and four-distractor conditions did not reach significance $[F(1,21)=3.80, p>.05]$. These results indicate that the presence of onset distractors increased the antisaccade cost when the target was premasked, but not when it was an onset.

Another ANOVA was conducted to compare the all-onset condition with the all-premask condition. Saccade latencies were longer in the all-premask than in the all-onset condition $[F(1,21)=124.29, p<.001]$ and antisaccade latencies were longer than prosaccade latencies $[F(1,21)=135.83$, $p<.001]$, but there was no significant interaction between condition and task $[F(1,21)=1.63, p>.20]$.

A further analysis was conducted to examine whether correct antisaccade latencies were affected by the presence of either an onset or a premasked digit at the antisaccade location. Note that only the display types with four or two onsets could be used for this analysis, since they were the only conditions with both onset and premasked distractors. The data of 3 participants were discarded because of an empty cell in at least one condition. An ANOVA on mean antisaccade latency with target type (onset vs. premask), display type (four onsets and two premasks vs. two onsets and four premasks), and antisaccade distractor (onset vs. premask) revealed a main effect of antisaccade distractor $[F(1,18)=19.61, p<.001]$. The mean antisaccade latency was $16 \mathrm{msec}$ longer when the distractor at the antisaccade location was premasked than when it was an onset. Furthermore, an interaction between display type and antisaccade distractor $[F(1,18)=8.62, p<.01]$ revealed that this effect was greater when there were four premasks and two onsets in the display (26-msec effect) than when there were two premasks and four onsets in the display (7-msec effect).

Intersaccade intervals. Finally, we examined the time it took participants to correct erroneous prosaccades and execute a subsequent antisaccade. This ISI represents the fixation time at (or near) the prosaccade location prior to the saccade toward the antisaccade location, for those trials on which the first saccade was directed toward the prosaccade location and the second toward the antisaccade location. Pooled across all conditions, $88.3 \%$ of trials with erroneous prosaccades were corrected by a subsequent saccade to the antisaccade location. In order to examine whether there were any differences in ISIs between conditions, we conducted an ANOVA on mean ISIs with target type (onset vs. premask) and display type (four onsets and two premasks vs. two onsets and four premasks) as factors. The data of 5 participants were discarded for this analysis because of at least one empty cell. No significant effects were found, but there was a nonsignificant trend toward longer ISIs in the condition with two onsets and four premasks $(129 \mathrm{msec})$ than in the condition with four onsets and two premasks $(115 \mathrm{msec})[F(1,16)=4.15, p>$ $.05]$. Furthermore, there was a nonsignificant trend toward longer ISIs with a premasked target $(127 \mathrm{msec})$ than with an onset target $(117 \mathrm{msec})[F(1,16)=3.58, p>.05]$.

\section{Discussion}

The results of Experiment 1 revealed that the attentional demands of the task affected the antisaccade cost. When the target was premasked, the antisaccade cost was greater than when the target was an onset. This finding is consistent with our previous findings (Godijn \& Kramer, 2006, 2007) that the antisaccade cost is greatest for the least salient target. Moreover, a novel finding of Experiment 1 was that when the target was premasked, the antisaccade cost was greater when onset distractors were presented than when no onset distractors were presented. Furthermore, when both onsets and premasks were presented, the proportion of erroneous prosaccades was greater when the target was premasked than when it was an onset. Finally, note that antisaccade generation was only impaired in the 
presence of distractors that received greater attentional priority than the target. There was no significant difference in the antisaccade costs between the all-onset and all-premask conditions, and when the target was an onset, the number of onset distractors did not affect the antisaccade cost. In fact, overall the number of onset distractors did not significantly affect the antisaccade cost. However, there was a nonsignificant trend in the premask target condition toward a greater antisaccade cost with two onset distractors than with four onset distractors. Although this finding was not statistically reliable and is not critical for our predictions, it is possible that the disruption caused by the onset distractors is greater when there are fewer of them. Specifically, there may be interference between multiple onsets to such an extent that the total level of disruption is decreased as the number of onsets increases. This idea is consistent with the results of Kramer, Cassavaugh, Irwin, Peterson, and Hahn (2001), who found that in the oculomotor capture task (see, e.g., Godijn \& Theeuwes, 2002; Theeuwes, Kramer, Hahn, \& Irwin, 1998; Theeuwes, Kramer, Hahn, Irwin, \& Zelinsky, 1999) oculomotor capture by task-irrelevant abrupt onsets was greater when a single onset was presented, rather than two.

To account for the results of Experiment 1, we propose that when the target is not an onset, attention is first allocated to onset distractors. Attention then needs to be disengaged from the onsets before the target can be localized. Thus, attentional capture by the onset distractors increased the attentional demands of target localization. We further propose that the attentional demands of target localization competed with the attentional selection of the antisaccade location, thereby increasing the antisaccade cost. Taken together, the results of Experiment 1 support the hypothesis that the attentional demands of the search task compete with the attentional selection of the antisaccade goal.

\section{EXPERIMENT 2}

In Experiment 2, another attentional manipulation was employed to examine whether attentional demands affect the antisaccade cost. Participants performed either an easy search (a numeral 2 among 7s) or a difficult search (a 2 among 5s). Classic visual search theories assume that an easy search is accomplished in parallel, whereas a difficult search is accomplished serially (e.g., Treisman \& Gelade, 1980; Wolfe, Cave, \& Franzel, 1989). However, since the serial versus parallel nature of visual search is disputed (e.g., Eckstein, Thomas, Palmer, \& Shimozaki, 2000; Palmer, Verghese, \& Pavel, 2000; Townsend, 1990), we simply refer to these search tasks as easy and difficult. However the processes underlying these search tasks are characterized, it is clear that the attentional demands of the difficult search task are greater than those of the easy one. Therefore, we predicted that the antisaccade cost would be greater in the difficult than in the easy search task. In addition, we varied the display size (two or six digits) in order to examine whether the number of display items affected the antisaccade cost and to verify our hypothesized difference in attentional demands between the two search tasks.
Finally, a pilot experiment had demonstrated that participants frequently scanned overtly for the target, especially in the difficult search condition. To prevent a high frequency of overt scanning behavior, we included $50 \%$ catch trials, on which no target was presented, and participants were required to keep their eyes on the fixation cross.

\section{Method}

Participants. After giving their informed consent, 24 students of the University of Illinois with normal or corrected-to-normal vision served as paid volunteers.

Stimuli, Procedure, and Design. The stimuli were the same as in the previous experiment, with the following exceptions. Either two or six digits were presented as onsets. When two digits were presented, they were always presented at opposite locations relative to the fixation cross. On half of the trials, a target digit (a 2) was presented together with distractor digits. On the other half of the trials (catch trials), all of the digits were distractors. Also, on half of the trials, the distractor digits were $5 \mathrm{~s}$ (difficult search), and on the other half, the distractor digits were $7 \mathrm{~s}$ (easy search). Participants performed four blocks of 192 trials, two prosaccade blocks and two antisaccade blocks. Each block was preceded by 20 practice trials. In prosaccade blocks, participants were instructed to execute a prosaccade toward the search target, and in the antisaccade blocks, they were instructed to execute an antisaccade away from the search target. On catch trials, they were required to keep their eyes on the fixation cross. All conditions were randomized within blocks, and the order of blocks was randomized across participants.

\section{Results}

Discarded data. Trials on which the initial saccade latency was below $100 \mathrm{msec}(6.8 \%)$ or above $1,200 \mathrm{msec}$ $(1.2 \%)$ were discarded from further analyses. In addition, $6.6 \%$ miss trials (on which the participants did not execute a saccade or on which the first saccade had an amplitude of less than $2^{\circ}$ ) were discarded from analyses. The participants executed $19.7 \%$ false alarms on catch trials.

Initial saccade destination. The distance between the initial saccade endpoint and the six possible object locations was used to determine the initial saccade destination. The saccade was assigned to the position closest to the saccade endpoint. Although there were no locations other than the prosaccade and antisaccade locations in display size two, this criterion enabled us to equate the required saccade accuracy between the two display sizes. Table 3 shows the percentages of initial saccades that were directed to the prosaccade location, the antisaccade location, and the "other" locations as a function of search task (easy vs. difficult), display size (two vs. six), and saccade task (pro- vs. antisaccade). An ANOVA on the percentage of erroneous prosaccades in the antisaccade task, with search task and display size as factors, revealed a significant effect of search task $[F(1,23)=12.75, p<.005]$. There were more erroneous prosaccades in the difficult than in the easy search task. There was no significant effect of display size on the percentage of erroneous prosaccades $[F(1,23)=2.51, p>.10]$, and there was no interaction between display size and search task $[F(1,23)<1]$. A further ANOVA was conducted on the percentage of erroneous saccades in the antisaccade task to the "other" locations. Here main effects of display size $[F(1,23)=$ 56.83, $p<.001]$ and search task $[F(1,23)=34.19, p<$ 
Table 3

Percentages of Initial Saccades to the Prosaccade Location (Pro), the Antisaccade Location (Anti), and Other Locations, As a Function of Search Task, Display Size, and Saccade Task in Experiment 2

\begin{tabular}{|c|c|c|c|c|c|c|c|}
\hline \multirow{2}{*}{$\begin{array}{c}\text { Search } \\
\text { Task }\end{array}$} & \multirow{2}{*}{$\begin{array}{c}\text { Display } \\
\text { Size }\end{array}$} & \multicolumn{3}{|c|}{ Prosaccade Task } & \multicolumn{3}{|c|}{ Antisaccade Task } \\
\hline & & Pro & Anti & Other & Pro & Anti & Other \\
\hline \multirow[t]{2}{*}{ Easy } & 2 & 98.6 & 0.5 & 0.9 & 5.5 & 93.8 & 0.7 \\
\hline & 6 & 98.6 & 0.3 & 1.1 & 8.6 & 89.4 & 2.0 \\
\hline \multirow[t]{2}{*}{ Difficult } & 2 & 94.6 & 4.5 & 0.9 & 11.6 & 87.7 & 0.7 \\
\hline & 6 & 80.9 & 2.8 & 16.3 & 12.8 & 74.8 & 12.4 \\
\hline
\end{tabular}

$.001]$ emerged, as well as an interaction of display size and search task $[F(1,23)=27.06, p<.001]$.

Saccade latencies. Table 4 shows the mean latencies of correct pro- and antisaccades and the antisaccade cost as a function of search task (easy vs. difficult) and display size (two vs. six). An ANOVA with search task, display size, and saccade task as within-subjects factors was conducted on the mean latencies of the correct saccades. A main effect of saccade task $[F(1,23)=37.83, p<.001]$ indicated that correct saccade latencies were longer in the antisaccade than in the prosaccade task. There were also main effects of search task $[F(1,23)=154.38, p<.001]$ and display size $[F(1,23)=255.72, p<.001]$. Saccade latencies were longer in the difficult than in the easy search task, and they were also longer with display size six than with display size two. Furthermore, there was an interaction between search task and display size $[F(1,23)=137.98$, $p<.001]$; that is, display size had a greater effect in the difficult search task than in the easy one. Finally, an interaction between saccade task and search task $[F(1,23)=$ $8.81, p<.01]$ indicated that the antisaccade cost was greater in the difficult than in the easy search task. The interaction between saccade task and display size did not reach significance $[F(1,23)=2.80, p>.10]$, nor did the three-way interaction between saccade task, search task, and display size $[F(1,23)<1]$.

Intersaccade intervals. Pooled across all conditions, $76.2 \%$ of trials with erroneous prosaccades were corrected by a subsequent saccade to the antisaccade location. The mean ISI was $169 \mathrm{msec}$. An ANOVA was conducted on mean ISIs, with search task (easy vs. difficult) and display size (two vs. six) as factors. The data of 5 participants were discarded from this analysis because of at least one empty cell. No significant effect emerged of either search task or display size $(F \mathrm{~S}<1)$, and the interaction between

Table 4

Latencies of Correct Saccades (in Milliseconds) in the Pro- and Antisaccade Tasks, As Well As the Antisaccade Cost, As a Function of Search Task and Display Size in Experiment 2

\begin{tabular}{|c|c|c|c|c|c|c|}
\hline \multirow{3}{*}{$\begin{array}{l}\text { Search } \\
\text { Task }\end{array}$} & \multirow{3}{*}{$\begin{array}{c}\text { Display } \\
\text { Size }\end{array}$} & \multicolumn{4}{|c|}{ Task } & \multirow{3}{*}{$\begin{array}{c}\text { Antisaccade } \\
\text { Cost }\end{array}$} \\
\hline & & \multicolumn{2}{|c|}{ Prosaccade } & \multicolumn{2}{|c|}{ Antisaccade } & \\
\hline & & $M$ & $S E$ & $M$ & $S E$ & \\
\hline \multirow[t]{2}{*}{ Easy } & 2 & 403 & 6.5 & 433 & 9.3 & 30 \\
\hline & 6 & 426 & 7.0 & 464 & 9.2 & 38 \\
\hline \multirow[t]{2}{*}{ Difficult } & 2 & 432 & 9.3 & 481 & 10.3 & 49 \\
\hline & 6 & 577 & 17.3 & 636 & 13.6 & 59 \\
\hline
\end{tabular}

search task and display size failed to reach significance $[F(1,18)=3.43, p>.05]$.

\section{Discussion}

The results of Experiment 2 were consistent with the hypothesis that the attentional demands of the search task affect the antisaccade cost. Specifically, the antisaccade cost was greater in the difficult search task (a 2 among 5s) than in the easy one (a 2 among 7s). The difficult search task also elicited more erroneous prosaccades than did the easy search task, although this finding is difficult to interpret, given the higher percentage of saccades to "other" locations in the difficult than in the easy search task. Display size had a large effect on saccade latencies, especially in the difficult search task, but it did not have a reliable effect on the antisaccade cost or on the percentage of erroneous prosaccades. Nevertheless, there were trends toward an effect of display size on performance. These findings will be addressed further in the General Discussion.

\section{GENERAL DISCUSSION}

The present study examined the effect of attentional demands on the antisaccade cost. Two attentional manipulations were employed: In Experiment 1, the search target and distractors were either premasked or presented as onsets. The results showed that the antisaccade cost was greater when the target was premasked than when the target was an onset. Furthermore, when the target was premasked, the antisaccade cost was greater when onset distractors were presented than when all distractors were premasked. In Experiment 2, we varied the difficulty of the search task and found that the antisaccade cost was greater in a difficult search task (a 2 among $5 \mathrm{~s}$ ) than in an easy one (a 2 among 7s). The results of both of these experiments indicate that the antisaccade cost increases as a function of the attentional demands of the search task.

\section{Antisaccade Cost and Attentional Demands}

Before addressing the effect of attentional demands on antisaccade generation, we will discuss the attentional mechanisms involved in the present search tasks. Classic models of visual search, such as the feature integration theory (e.g., Treisman \& Gelade, 1980) and guided search (e.g., Wolfe et al., 1989), have proposed that visual search consists of two stages: a preattentive stage, in which parallel processing of basic features is accomplished, and an attentive stage, in which attention is allocated serially for more complex processing (e.g., conjunctions of features). Indeed, in some search tasks, performance is not (or is hardly) affected by the number of distractors, but in others, response times increase substantially with each additional distractor. These tasks quickly became labeled as "parallel" or "serial" search tasks (e.g., Treisman \& Gelade, 1980). However, several issues in visual search, such as the nature of preattentive processing and the degree to which search is parallel or serial, are currently disputed (see Wolfe, 2003, for a review). Nevertheless, it is clear that some search tasks place greater attentional demands. In some tasks, the target appears to pop out from among the distractors, irrespec- 
tive of how many distractors are presented (hence, response times are more or less independent of display size). For example, in Experiment 2 of the present study, prosaccade latencies in the easy search task increased by a mere $6 \mathrm{msec}$ per extra distractor. It is thus clear that parallel processing of the digits in this search task is sufficient to localize the target. In contrast, in the difficult search task, prosaccade latencies increased by about $36 \mathrm{msec}$ per extra distractor. Therefore, there can be no doubt that the difficult search task was attentionally more demanding.

In Experiment 1, the search task was the same as the easy search task of Experiment 2. However, attentional demands were manipulated by varying the number of premasked and onset digits. Research has shown that onsets capture attention and therefore receive prioritized processing (e.g., Theeuwes, 1991; Yantis, 1996). Thus, when the target is premasked and onset distractors are presented, attention is first allocated to the distractors. This is consistent with the results of Experiment 1, in which correct prosaccade latencies when both premasked and onset digits were presented were about 45 msec shorter when the target was an onset rather than premasked. It may seem somewhat surprising that the presence of onset distractors had no effect on prosaccade latencies to premasked targets. However, in addition to the attentional capture by the onsets, it is likely that they have an alerting effect, in that they indicate the presence of the target in the display. Gibson and Kelsey (1998) provided evidence that salient display features such as onsets capture attention when they signal the presence of the target. Thus, in our Experiment 1, the onset distractors may have had two opposite effects on correct prosaccade latencies. First, they increased saccade latencies, because the wrong digits received attentional priority. Second, they decreased saccade latencies, because they alerted participants to the presence of the target.

As in the easy search task of Experiment 2, we can assume that in the all-premask condition of Experiment 1, the target could be localized while attention was allocated in parallel between the digits (i.e., the easy search task was not disrupted by any onsets). However, when onset distractors were presented, one or more of these onsets received attentional priority. In order to localize the target, attention needed to be disengaged from the onset distractors and reallocated at the target location. In other words, the presentation of onset distractors increased the attentional demands of the task.

The results of both experiments provided evidence that increasing the attentional demands increased the antisaccade cost. That is, the attentional demands influenced antisaccade latencies to a greater extent than prosaccade latencies. Thus, the attentional demands did not merely increase the time needed for localization of the target, which would have increased both prosaccade and antisaccade latencies equally. We propose that the attentional demands of target localization compete with those of selecting the antisaccade location. It is important to note that the antisaccade cost is not simply affected by any manipulation that affects prosaccade latencies, and that some manipulations that do not affect prosaccade latencies do affect the antisaccade cost. First, although onset distractors had no effect on prosaccade latencies toward premasked targets in Experiment 1, they did have a significant effect on antisaccade latencies. Second, although Experiment 2 featured a very robust effect of display size on prosaccade latencies, the effect of display size was not statistically greater in the antisaccade than in the prosaccade task. There was a nonsignificant trend toward an interaction between display size and saccade task, but it is possible that the display size manipulation affected attention in a way fundamentally different from our other attentional manipulations. Specifically, although increasing the display size does increase the time needed for target location, this likely does not have an effect on the nature of attentional allocation. For example, it is likely that target localization requires more attentional resources at the target location in the difficult than in the easy condition, irrespective of the number of distractors in the display. Similarly, when onset distractors capture attention in the easy search task, the subsequent deallocation of attention from the onsets and its reallocation at the target location may result in more attentional resources being directed at the target location than in conditions without onset distractors (in which parallel attentional allocation may be sufficient for target localization). Indeed, we propose that attentional resources allocated at the prosaccade location compete with the attentional resources required for the selection of the antisaccade goal. This idea will be further discussed in subsequent sections, but first, we will address the prosaccade errors in the antisaccade task.

\section{Prosaccade Errors}

In the present study, the percentage of erroneous prosaccades in the antisaccade task typically followed the pattern of results of the antisaccade cost. Specifically, when both onsets and premasks were presented, the percentage of erroneous prosaccades was greater when the target was premasked than when it was an onset. The percentage of erroneous prosaccades was also greater in the difficult than in the easy search task. However, these results should be interpreted cautiously, because the conditions in which a high percentage of erroneous prosaccades were generated also elicited more saccades to "other" locations (i.e., not to the pro- or antisaccade location). Nevertheless, it seems unlikely that the difference in percentages of erroneous prosaccades is completely due to a higher percentage of guesses in these conditions. Moreover, previous studies also revealed that manipulations that affect the antisaccade cost also typically affect the percentage of erroneous prosaccades. For example, previous research has shown that the antisaccade cost and the percentage of erroneous prosaccades are both greater for color singleton targets than for onset or offset targets, and that both are also greater for onset than for offset targets (Godijn \& Kramer, 2007). Furthermore, when pro- and antisaccade instructions are mixed within blocks, the percentage of erroneous prosaccades and the antisaccade cost are both negatively related to the relative frequency of antisaccade trials (Massen, 2004). Both are also negatively affected when a working memory load is added in a dual-task study (see, 
e.g., Roberts, Hager, \& Heron, 1994). They both decrease as a function of age from ages 8 to 18 (Kramer, Gonzalez de Sather, \& Cassavaugh, 2005), and patients with frontallobe damage show deficits in terms of both erroneous prosaccades and the antisaccade cost (e.g., Guitton et al., 1985 ). Thus, finding a similar pattern of results in the percentage of erroneous prosaccades and in the antisaccade cost is consistent with a number of previous studies.

The erroneous-prosaccade findings of the present study, as well as previous findings from our lab (Godijn \& Kramer, 2006, 2007), are inconsistent with the idea that prosaccades are reflexive. For example, in Experiment 1, erroneous prosaccades occurred on $26 \%-27 \%$ of trials in the antisaccade task when the target was premasked and onset distractors were presented. Thus, even though salient distractors were present that captured attention, the eyes still moved to the prosaccade location on a high percentage of trials. Moreover, we recently demonstrated that the percentage of erroneous prosaccades was greater for static targets (i.e., uniquely unchanging targets) than for dynamic ones (i.e., unique onsets and offsets). These findings suggest that there is an endogenous component to erroneous prosaccades (e.g., Godijn \& Kramer, 2006). Specifically, we propose that salient objects (in particular, onsets; e.g., Yantis, 1996) capture attention exogenously, but that endogenous factors play an important role in determining whether this attentional capture is followed by a saccade to the same location. Indeed, previous research has demonstrated that separate transient and sustained components are involved in visual attention (e.g., Most, Scholl, Clifford, \& Simons, 2005; Müller \& Rabbitt, 1989; Nakayama \& Mackeben, 1989). The transient component corresponds to exogenous attention, since it is independent from voluntary control and results in rapid attentional capture of short duration; the sustained component corresponds to endogenous attention, since it has a longer time course and is under voluntary control (e.g., Nakayama \& Mackeben, 1989). Moreover, Most et al. proposed that the degree to which attention is sustained (i.e., sustained or endogenous attention) on a specific object that has captured attention (i.e., transient or exogenous attention) depends on the top-down task set. That is, if the object that has captured attention is task-relevant, attention will be sustained at that location. Similarly, Theeuwes, Atchley, and Kramer (2000; see also Theeuwes \& Godijn, 2001) proposed that salient singletons capture attention exogenously, but the speed of attentional disengagement depends on the attentional control settings. Thus, attention dwells longer on objects that to some degree match the attentional control settings than on objects that do not in any way.

This distinction between transient and sustained attention appears to be very helpful in understanding the endogenous component of erroneous prosaccades. The prosaccade stimulus is task-relevant, since it determines the location of the saccade goal. If the prosaccade stimulus captures attention exogenously, one might then expect attention to be sustained at that location endogenously because of its task relevance. This in turn increases the probability that an erroneous saccade will be directed toward the prosaccade location (e.g., Godijn \& Kramer, 2006, 2007).
It might be argued that this endogenous component is relevant only for complex visual search tasks such as those in the present study, but not in the simple standard antisaccade task, in which only a single stimulus is presented (see, e.g., Fischer \& Weber, 1992; Hallett, 1978). Indeed, it cannot be disputed that the attentional demands of localizing the prosaccade stimulus are greater in the present visual search tasks. However, there is reason to believe that even in the simple standard antisaccade task, the relevance of the prosaccade stimulus plays an important role. For example, Walker et al. (1998) demonstrated in a single-case study that their frontal-lobe patient executed a high percentage of erroneous prosaccades toward the peripheral onset, consistent with previous research. However, when this patient was required to remain fixated and to ignore the peripheral onset, he was able to do so quite well. Thus, the task relevance of the peripheral onset had an effect on the patient's ability to refrain from executing a saccade in its direction. According to our proposed role of attention in antisaccade generation, the onset exogenously elicited a transient shift of attention in both the fixation and the antisaccade task. However, only in the antisaccade task was attention subsequently sustained endogenously, due to the task relevance of the onset. We further propose that this endogenous attentional component at the prosaccade location competes with the endogenous allocation of attention at the antisaccade location.

\section{Competition Account of Antisaccade Generation}

Several models of oculomotor control have been developed in which the saccade goal is selected by means of competitive interactions in a spatial selection map (e.g., Findlay \& Walker, 1999; Godijn \& Theeuwes, 2002; Kopecz, 1995; Trappenberg, Dorris, Munoz, \& Klein, 2001). Lateral inhibition from one location on another implies that the greater the activation at one location, the more the other location is inhibited. If we apply this logic to the antisaccade task, we can assume that activating the prosaccade location results in lateral inhibition of the antisaccade location. Competition models of oculomotor control do not all agree on the temporal aspects of saccade target selection. Some models (e.g., Godijn \& Theeuwes, 2002; Trappenberg et al., 2001) assume that a saccade is generated once an activation threshold is reached at one location in the spatial selection map, but others (e.g., Findlay \& Walker, 1999) assume that a separate fixation system competes with the move system (the spatial selection map) and that a saccade is generated once the activation in the fixation system falls beneath a certain threshold level. However, the difference between these models isn't as large as it would appear, since the former models assume that the fixation location is part of the spatial selection map and that the activation level at the fixation location is an important factor in saccade generation. That is, activation of the fixation location laterally inhibits other locations, so that a strongly activated fixation location can prevent other locations from reaching threshold.

It should be noted that these competition models are models of oculomotor control, which may seem to distinguish them from our proposed competition account of 
antisaccade generation, in which attentional allocation plays a crucial role. However, competitive interactions are also an important factor in attentional control (see, e.g., Desimone \& Duncan, 1995; Itti \& Koch, 2000), and it is well-known that attention and saccades are strongly related (e.g., Deubel \& Schneider, 1996; Hoffman \& Subramaniam, 1995; Kowler et al., 1995). For example, Deubel and Schneider proposed that a common selection mechanism underlies both object recognition and (oculo)motor control. Although there may well be a distinction between oculomotor processes and premotor or attentional processes (e.g., Juan, Shorter-Jacobi, \& Schall, 2004; Klein \& Pontefract, 1994; Sato \& Schall, 2003), for simplicity's sake we will assume a single spatial selection map, in which locations interact laterally and thereby compete for selection of the saccade goal. Thus, when attention is directed at the prosaccade location in order to localize the target, the antisaccade location is laterally inhibited; the more attentional resources are directed at the prosaccade location, the stronger the activation at that location becomes, and the more the antisaccade location is laterally inhibited. If the prosaccade stimulus is an onset, attention is captured by the onset, and the prosaccade location is transiently activated in the spatial selection map. Indeed, for Trappenberg et al. (2001), this exogenous component of the onset at the prosaccade location was modeled as a transient activation at that location. On the basis of the present findings and of previous findings from our lab (Godijn \& Kramer, 2006, 2007), we propose that attention may be sustained at the prosaccade location endogenously. Thus, the activation level in the spatial selection map remains strong as long as attention is sustained at the prosaccade location. In order to generate an antisaccade, an attentional shift from the prosaccade location to the antisaccade location is required. The competitive integration models of Trappenberg et al. (2001) and Godijn and Theeuwes (2002) assume that a saccade is generated once an activation threshold is reached. Thus, when the localization of the search target is attentionally more demanding, the prosaccade location is activated more strongly and longer, thereby increasing the probability that a threshold will be reached at the prosaccade location. Likewise, the Findlay and Walker (1999) model assumes that a saccade is generated once activation in the fixation system is below a threshold level of activation. At that moment, a saccade is generated toward the location with the greatest activation in the spatial selection map. The greater the activation at the prosaccade location and the longer this activation is sustained, the greater the probability that a saccade will be generated toward that location. Moreover, the longer that activation is sustained at the prosaccade location, the more time will be required for the activation level at the antisaccade location to surpass the level at the prosaccade location, thus increasing the antisaccade cost.

A competition account of antisaccade generation has also been proposed by Kristjánsson and colleagues to account for their findings (Kristjánsson, 2007; Kristjánsson et al., 2001; Kristjánsson et al., 2004). For example, Kristjánsson et al. (2001) found that a secondary peripheral discrimination task resulted in shorter antisaccade latencies. To account for this counterintuitive finding, they proposed that the secondary task interfered with the reflexive prosaccade, thereby giving the antisaccade location a competitive edge relative to the prosaccade location. Although our view is similar, since we agree that competitive interactions occur between the prosaccade and antisaccade locations, we disagree that the secondary task interferes with the reflexive, or transient, component related to the prosaccade. Instead, according to our view, the attentionally demanding secondary task interferes with the sustained component at the prosaccade location. That is, in the standard simple antisaccade task, in which a single onset is presented as the prosaccade stimulus, a transient, exogenous component of attention is followed by a sustained, endogenous component because of the task relevance of the stimulus. However, since localization of the single stimulus is relatively straightforward, this endogenous component is likely to be counterproductive, since it competes with attentional allocation at the antisaccade location. Thus, preventing attention from being sustained endogenously at the prosaccade location facilitates the antisaccade generation. It should be clear that this points to a significant difference between the standard simple antisaccade task and the search tasks in the present study. Specifically, in some conditions of the present study, sustaining attention at the prosaccade stimulus was to a certain extent crucial for localizing the search target, because letter identity needed to be processed. Although this enhanced the activation level of the prosaccade location in the spatial selection map, resulting in strong competition against the antisaccade location, this was a necessary part of the antisaccade task, particularly in the attentionally demanding search tasks.

\section{Neural Correlates of Competition in the Oculomotor System}

The competition model of antisaccade generation is consistent with neurophysiological studies that have provided evidence for competitive interactions in brain regions involved in oculomotor control, such as the superior colliculus (e.g., Munoz \& Fecteau, 2002; Munoz \& Istvan, 1998; Munoz \& Wurtz, 1993). These studies have also demonstrated the importance of fixation neurons, which laterally inhibit activity of saccade-related neurons (e.g., Munoz \& Wurtz, 1993). Furthermore, Everling, Dorris, Klein, and Munoz (1999) showed that the activity of fixation-related cells was enhanced prior to the appearance of the target on antisaccade trials relative to prosaccade trials.

The difference between pro- and antisaccade trials in neural activity prior to the appearance of the target indicates that pretarget preparation plays an important role in antisaccade generation. Indeed, the importance of preparing for the antisaccade task prior to the appearance of the target has been well-established in several recent fMRI studies, which have revealed differences in neural activation prior to the appearance of the target between the proand antisaccade tasks (e.g., Connolly, Goodale, Menon, \& Munoz, 2002; Curtis \& D’Esposito, 2003; DeSouza, Menon, \& Everling, 2003; Ford, Goltz, Brown, \& Everling, 2005). For example, Ford et al. showed that neural 
activation was greater on correct antisaccade trials than on correct prosaccade trials prior to appearance of the target in several frontal and parietal areas. Furthermore, the preparatory activation of the frontal areas was also greater on correct antisaccade trials than on erroneous prosaccade trials in the antisaccade task.

Taken together, these findings suggest that oculomotor inhibition is involved in the antisaccade task. However, this inhibition appears to inhibit oculomotor activity generally, rather than any specific saccade program that is set up well before the target is presented. This is contrary to the traditional view of a saccade program that runs until an inhibitory or cancellation signal is given (e.g., Guitton et al., 1985; Hallett \& Adams, 1980). Nevertheless, these findings do indicate that part of the antisaccade cost might be caused by a difference in the degree to which the fixation system is activated in the prosaccade task relative to the antisaccade task.

\section{AUTHOR NOTE}

Correspondence relating to this article may be sent to R. Godijn, Department of Cognitive Psychology, Vrije Universiteit Amsterdam, Van der Boechorststraat 1, 1081 BT Amsterdam, The Netherlands (e-mail: rj.godijn@psy.vu.nl).

\section{REFERENCES}

Connolly, J. D., Goodale, M. A., Menon, R. S., \& Munoz, D. P. (2002). Human fMRI evidence for the neural correlates of preparatory set. Nature Neuroscience, 5, 1345-1352.

Curtis, C. E., \& D’Esposito, M. (2003). Success and failure suppressing reflexive behavior. Journal of Cognitive Neuroscience, $\mathbf{1 5}$, 409-418.

Desimone, R., \& Duncan, J. (1995). Neural mechanisms of selective visual attention. Annual Review of Neuroscience, 18, 193-222.

DeSouza, J. F. X., Menon, R. S., \& Everling, S. (2003). Preparatory set associated with pro-saccades and anti-saccades in humans investigated with event-related fMRI. Journal of Neurophysiology, 89, 1016-1023.

Deubel, H., \& Schneider, W. X. (1996). Saccade target selection and object recognition: Evidence for a common attentional mechanism. Vision Research, 36, 1827-1837.

Eckstein, M. P., Thomas, J. P., Palmer, J., \& Shimozaki, S. S. (2000) A signal detection model predicts the effects of set size on visual search accuracy for feature, conjunction, triple conjunction, and disjunction displays. Perception \& Psychophysics, 62, 425-451.

Everling, S., Dorris, M. C., Klein, R. M., \& Munoz, D. P. (1999). Role of primate superior colliculus in preparation and execution of antisaccades and pro-saccades. Journal of Neuroscience, 19, 2740-2754.

EverLing, S., \& MunOz, D. P. (2000). Neuronal correlates for preparatory set associated with pro-saccades and anti-saccades in the primate frontal eye field. Journal of Neuroscience, 20, 387-400.

FINDLAY, J. M., \& WALKER, R. (1999). A model of saccade generation based on parallel processing and competitive inhibition. Behavioral \& Brain Sciences, 22, 661-721.

Fischer, B., \& WeBer, H. (1992). Characteristics of "anti" saccades in man. Experimental Brain Research, 89, 415-424.

Ford, K. A., Goltz, H. C., Brown, M. R. G., \& Everling, S. (2005) Neural processes associated with antisaccade task performance investigated with event-related fMRI. Journal of Neurophysiology, 94, 429-440.

Gibson, B. S., \& Kelsey, E. M. (1998). Stimulus-driven attentional capture is contingent on attentional set for displaywide visual features. Journal of Experimental Psychology: Human Perception \& Performance, 24, 699-706.

Godisn, R., \& Kramer, A. F. (2006). Prosaccades and antisaccades to onsets and color singletons: Evidence that erroneous prosaccades are not reflexive. Experimental Brain Research, 172, 439-448.
Godisn, R., \& Kramer, A. F. (2007). Antisaccade costs with static and dynamic targets. Perception \& Psychophysics, 69, 802-815.

GodiJn, R., \& Pratt, J. (2002). Endogenous saccades are preceded by shifts of visual attention: Evidence from cross-saccadic priming effects. Acta Psychologica, 110, 83-102.

Godis, R., \& Theeuwes, J. (2002). Programming of endogenous and exogenous saccades: Evidence for a competitive integration model. Journal of Experimental Psychology: Human Perception \& Performance, 28, 1039-1054.

Guitton, D., Buchtel, H. A., \& Douglas, R. M. (1985). Frontal lobe lesions in man cause difficulties in suppressing reflexive glances and in generating goal-directed saccades. Experimental Brain Research, 58, 455-472.

Hallett, P. E. (1978). Primary and secondary saccades to goals defined by instructions. Vision Research, 18, 1279-1296.

Hallett, P. E., \& Adams, B. D. (1980). The predictability of saccade latency in a novel voluntary oculomotor task. Vision Research, 20, 329-339.

Hoffman, J. E., \& Subramaniam, B. (1995). The role of visual attention in saccadic eye movements. Perception \& Psychophysics, 57, 787-795.

IтTі, L., \& КосH, C. (2000). A saliency-based search mechanism for overt and covert shifts of visual attention. Vision Research, 40, 1489-1506.

Juan, C. H., Shorter-JACobi, S. M., \& Schall, J. D. (2004). Dissociation of spatial attention and saccade preparation. Proceedings of the National Academy of Sciences, 101, 15541-15544.

Klein, R. M., \& Pontefract, A. (1994). Does oculomotor readiness mediate cognitive control of visual attention? Revisited! In C. Umiltà \& M. Moscovitch (Eds.), Attention and performance XV: Conscious and nonconscious information processing (pp. 333-350). Cambridge, MA: MIT Press.

Kopecz, K. (1995). Saccadic reaction times in gap/overlap paradigms: A model based on integration of intentional and visual information on neural, dynamic fields. Vision Research, 35, 2911-2925.

Kowler, E., Anderson, E., Dosher, B., \& Blaser, E. (1995). The role of attention in the programming of saccades. Vision Research, 35, 1897-1916.

Kramer, A. F., Cassavaugh, N. D., Irwin, D. E., Peterson, M. S., \& HaHN, S. (2001). Influence of single and multiple onset distractors on visual search for singleton targets. Perception \& Psychophysics, 63, 952-968.

Kramer, A. F., Gonzalez de Sather, J. C. M., \& Cassavaugh, N. D. (2005). Development of attentional and oculomotor control. Developmental Psychology, 41, 760-772.

KRISTJÁnSSON, Á. (2007). Saccade landing point selection and the competition account of pro- and antisaccade generation: The involvement of visual attention-A review. Scandinavian Journal of Psychology, 48, 97-113

Kristuánsson, Á., Chen, Y., \& NaKayama, K. (2001). Less attention is more in the preparation of antisaccades, but not prosaccades. Nature Neuroscience, 4, 1037-1042.

Kristjánsson, Á., Vandenbroucke, M. W., \& Driver, J. (2004). When pros become cons for anti- versus prosaccades: Factors with opposite or common effects on different saccade types. Experimental Brain Research, 155, 231-244.

Massen, C. (2004). Parallel programming of exogenous and endogenous components in the antisaccade task. Quarterly Journal of Experimental Psychology, 57A, 475-498.

Milea, D., Lehéricy, S., Rivaud-Péchoux, S., Duffau, H., Lobel, E., CAPelle, L., ET AL. (2003). Antisaccade deficit after anterior cingulate cortex resection. NeuroReport, 14, 283-287.

Most, S. B., Scholl, B. J., Clifford, E. R., \& Simons, D. J. (2005). What you see is what you set: Sustained inattentional blindness and the capture of awareness. Psychological Review, 112, 217-242.

Müller, H. J., \& RabBitT, P. M. A. (1989). Reflexive and voluntary orienting of visual attention: Time course of activation and resistance to interruption. Journal of Experimental Psychology: Human Perception \& Performance, 15, 315-330.

Munoz, D. P., \& Everling, S. (2004). Look away: The anti-saccade task and the voluntary control of eye movement. Nature Reviews Neuroscience, 5, 218-228.

Munoz, D. P., \& Fecteau, J. H. (2002). Vying for dominance: Dynamic 
interactions control visual fixation and saccadic initiation in the superior colliculus. Progress in Brain Research, 140, 3-19.

Munoz, D. P., \& IstVAn, P. J. (1998). Lateral inhibitory interactions in the intermediate layers of the monkey superior colliculus. Journal of Neurophysiology, 79, 1193-1209.

Munoz, D. P., \& WurTz, R. H. (1993). Fixation cells in monkey superior colliculus: II. Reversible activation and deactivation. Journal of Neurophysiology, 70, 576-589.

NaKayAma, K., \& Mackeben, M. (1989). Sustained and transient components of focal visual attention. Vision Research, 29, 1631-1647.

OlK, B., \& Kingstone, A. (2003). Why are antisaccades slower than prosaccades? A novel finding using a new paradigm. NeuroReport, 14, 151-155.

Palmer, J., Verghese, P., \& Pavel, M. (2000). The psychophysics of visual search. Vision Research, 40, 1227-1268.

Pierrot-Deseilligny, C., Ploner, C. J., Müri, R. M., Gaymard, B., \& RivaUd-PÉcHOUX, S. (2002). Effects of cortical lesions on saccadic eye movements in humans. In H. J. Kaminski \& R. J. Leigh (Eds.), Neurobiology of eye movements: From molecules to behavior (Annals of the New York Academy of Sciences, Vol. 956, pp. 216-229). New York: New York Academy of Sciences.

Pierrot-Deseilligny, C., Rivaud, S., Gaymard, B., \& Agid, Y (1991). Cortical control of reflexive visually-guided saccades. Brain, 114, 1473-1485.

Pratt, J., \& Trottier, L. (2005). Pro-saccades and anti-saccades to onset and offset targets. Vision Research, 45, 765-774.

Roberts, R. J., Jr., Hager, L. D., \& Heron, C. (1994). Prefrontal cognitive processes: Working memory and inhibition in the antisaccade task. Journal of Experimental Psychology: General, 123, 374-393.

SAto, T. R., \& Schall, J. D. (2003). Effects of stimulus-response compatibility on neural selection in frontal eye field. Neuron, 38, 637-648.

THEeuwes, J. (1991). Exogenous and endogenous control of attention: The effect of visual onsets and offsets. Perception \& Psychophysics, 49, 83-90.

Theeuwes, J., Atchley, P., \& Kramer, A. F. (2000). On the time course of top-down and bottom-up control of visual attention. In S. Monsell \& J. Driver (Eds.), Control of cognitive processes: Attention and performance XVIII (pp. 105-124). Cambridge, MA: MIT Press.

Theeuwes, J., \& Godisn, R. (2001). Attentional and oculomotor cap- ture. In C. L. Folk \& B. S. Gibson (Eds.), Attraction, distraction and action: Multiple perspectives on attentional capture (pp. 121-149). New York: Elsevier.

Theeuwes, J., Kramer, A. F., Hahn, S., \& Irwin, D. E. (1998). Our eyes do not always go where we want them to go: Capture of the eyes by new objects. Psychological Science, 9, 379-385.

Theeuwes, J., Kramer, A. F., Hahn, S., Irwin, D. E., \& Zelinsky, G. J. (1999). Influence of attentional capture on oculomotor control. Journal of Experimental Psychology: Human Perception \& Performance, 25, 1595-1608.

Townsend, J. T. (1990). Serial vs. parallel processing: Sometimes they look like Tweedledum and Tweedledee but they can (and should) be distinguished. Psychological Science, 1, 46-54.

Trappenberg, T. P., Dorris, M. C., Munoz, D. P., \& Klein, R. M. (2001). A model of saccade initiation based on the competitive integration of exogenous and endogenous signals in the superior colliculus. Journal of Cognitive Neuroscience, 13, 256-271.

Treisman, A. M., \& Gelade, G. (1980). A feature-integration theory of attention. Cognitive Psychology, 12, 97-136.

Walker, R., Husain, M., Hodgson, T. L., Harrison, J., \& KenNARD, C. (1998). Saccadic eye movement and working memory deficits following damage to human prefrontal cortex. Neuropsychologia, 36, 1141-1159.

WoLfE, J. M. (2003). Moving towards solutions to some enduring controversies in visual search. Trends in Cognitive Sciences, 7, 70-76.

Wolfe, J. M., Cave, K. R., \& Franzel, S. L. (1989). Guided search: An alternative to the feature integration model for visual search. Journal of Experimental Psychology: Human Perception \& Performance, 15, 419-433.

Wolfe, J. M., \& Horowitz, T. S. (2004). What attributes guide the deployment of visual attention and how do they do it? Nature Reviews Neuroscience, 5, 495-501.

YAntis, S. (1996). Attentional capture in vision. In A. F. Kramer, M. G. H. Coles, \& G. D. Logan (Eds.), Converging operations in the study of visual selective attention (pp. 45-76). Washington, DC: American Psychological Association.

(Manuscript received March 15, 2007; revision accepted for publication January 18, 2008.) 